\title{
Universiteit
}

Leiden

The Netherlands

\section{Rotational resonance NMR of C-13(2)-labelled retinal: quantitative internuclear distance determination}

Feng, X.; Verdegem, P.J.E.; Lee, Y.K.; Helmle, M.; Shekar, S.C.; Groot, H.J.M. de; ... ; Levitt, M.H.

\section{Citation}

Feng, X., Verdegem, P. J. E., Lee, Y. K., Helmle, M., Shekar, S. C., Groot, H. J. M. de, ... Levitt, M. H. (2002). Rotational resonance NMR of C-13(2)-labelled retinal: quantitative internuclear distance determination. Solid State Nuclear Magnetic Resonance, 14(2), 81-90. doi:10.1016/S0926-2040(99)00019-3

Version:

Publisher's Version

License:

Licensed under Article 25fa Copyright Act/Law (Amendment Taverne)

Downloaded from: $\quad \underline{\text { https://hdl.handle.net/1887/3239373 }}$

Note: To cite this publication please use the final published version (if applicable). 


\title{
Rotational resonance $\mathrm{NMR}$ of ${ }^{13} \mathrm{C}_{2}$-labelled retinal: quantitative internuclear distance determination
}

\author{
X. Feng a,1, P.J.E. Verdegem ${ }^{\text {b,2 }}$, Y.K. Lee ${ }^{\mathrm{a}, 3}$, M. Helmle ${ }^{\mathrm{a}, 4}$, S.C. Shekar ${ }^{\text {a,5, }}$, \\ H.J.M. de Groot ${ }^{\mathrm{b}}$, J. Lugtenburg ${ }^{\mathrm{b}}$, M.H. Levitt ${ }^{\mathrm{a}, *}$ \\ a Physical Chemistry Division, Arrhenius Laboratory, Stockholm University, Stockholm S-106 91, Sweden \\ ${ }^{\mathrm{b}}$ Leiden Institute of Chemistry, Gorlaeus Laboratories, Leiden University, NL 2300 RA Leiden, Netherlands
}

Received 10 March 1999; accepted 21 April 1999

\begin{abstract}
Rotational resonance phenomena are investigated in the solid-state magic-angle spinning NMR of all- $E-\left[11,20-{ }^{13} \mathrm{C}_{2}\right]$-retinal at a magnetic field of $4.7 \mathrm{~T}$. We find good agreement between experiments and numerical simulations for the rotational resonance spectral peakshapes and for the rotor-driven magnetization exchange. The internuclear distance between the ${ }^{13} \mathrm{C}$-labelled C11 and C20 sites is determined to be $0.301 \pm 0.008 \mathrm{~nm}$ (from rotational resonance spectra) and $0.300 \pm 0.010$ $\mathrm{nm}$ (from rotor-driven magnetization exchange), in agreement with the X-ray crystallographic distance of $0.296 \mathrm{~nm}$. We show rotational resonance spectra which display perturbations from intermolecular homonuclear spin-spin interactions. (C) 1999 Elsevier Science B.V. All rights reserved.
\end{abstract}

Keywords: Rotational resonance NMR; Internuclear distance; ${ }^{13} \mathrm{C}_{2}$-labelled retinal

\section{Introduction}

Solid-state NMR may be used to determine molecular structure in systems which are difficult or

\footnotetext{
* Corresponding author. Fax: +46-8-152187; E-mail: mhl@physc.su.se

${ }^{1}$ Present address: ABB Corporate Research, Västerås, S-721 78, Sweden.

${ }^{2}$ Present address: Department of Radiology, Academic Hospital Nijmegen, 6500 HB Nijmegen, Netherlands.

${ }^{3}$ Present address: Quantum Magnetics, 740 Kenamar Court, San Diego, CA 92121-2425, USA.

${ }^{4}$ Present address: Department of Solid-State NMR Spectroscopy, Max-Planck-Institute for Biochemistry, D-82152 Martinsried, Germany.

${ }^{5}$ Present address: Department of Chemistry, University of Louisville, Louisville, KY 40292, USA.
}

impossible to crystallize, such as many polymers, biological molecules, and surface species. Molecular structural information is obtained from the magnitudes of spin-spin interactions, such as through-space internuclear dipolar couplings [1-4], or chemical shift tensors [5-7]. It is also possible to extract molecular structural information from the relative or absolute orientations of spin interaction tensors [8$10]$.

The magnitude of the through-space dipole-dipole couplings between nuclear spins is a direct source of molecular structural information, because it only depends on fundamental constants and the inverse third power of the internuclear distance. However, these couplings are quite difficult to access in the context of high-resolution solid-state NMR. This 
is because magic-angle spinning (MAS) is normally used to enhance the sensitivity and the spectral resolution, by suppressing the effect of the chemical shift anisotropies. Unfortunately, MAS also tends to average out the structurally informative through-space dipole-dipole couplings.

A variety of methods have been proposed for retrieving the dipolar coupling information in the presence of magic-angle spinning. One of the simplest is rotational resonance (RR) $[1,3,11,12]$. Consider a sample containing dilute homonuclear spin pairs, for example, ${ }^{13} \mathrm{C}$ pairs deliberately introduced by isotopic labelling. Rotational resonance occurs when the sample rotation frequency $\omega_{\mathrm{r}}$ matches the condition $\omega_{\Delta}^{\text {iso }}=n \omega_{\mathrm{r}}$, where $\omega_{\Delta}^{\text {iso }}$ is the difference in isotropic chemical shift frequencies of the paired spins, and $n$ is a small integer denoting the order of the rotational resonance. There are two main consequences of the reintroduction of the through-space dipolar coupling. First, the normally narrow spectral peaks acquire splittings and broadenings; Second, there is rotor-driven exchange of longitudinal magnetization between the spin sites. These two effects provide alternative routes for the estimation of the dipolar spin-spin couplings, and hence internuclear distances $[1,3]$.

So far, the exchange of longitudinal magnetization has been most widely used for biomolecular structural investigations [13-24]. This requires a two-dimensional experimental procedure, since longitudinal magnetization cannot be observed directly. The direct observation of spectral peakshapes at rotational resonance is potentially much faster [25], but has been relatively less explored for structural studies. Most quantitative treatments of rotational resonance spectra have been on compounds with short ${ }^{13} \mathrm{C}-{ }^{13} \mathrm{C}$ distances [1,3], although McDermott et al. reported the determination of an internuclear distance of about $0.27 \mathrm{~nm}$ between two ${ }^{31} \mathrm{P}$ spins [13].

Although most of the reported spectral peakshapes conform to theoretical expectations, there are also several reports which are in conflict with current theory. For example, Peersen et al. showed rotational resonance spectral peakshapes for pairs of ${ }^{13} \mathrm{C}$ nuclei separated by $0.37 \mathrm{~nm}$ in a small peptide, which do not conform to the present rotational resonance theory [22]. Rather similar observations were made by some of us on a set of ${ }^{13} \mathrm{C}_{2}$-labelled retinals at a magnetic field of $9.4 \mathrm{~T}$ [25]. In the latter work, the observed peaks typically had a very different form for the two sites, with one peak split and the other unsplit, whereas available theory always predicts a more-or-less symmetrical splitting at rotational resonance. Despite these striking discrepancies, it still proved possible to obtain quantitative distance estimations from the split peak by a semi-empirical calibration procedure [25]. In a forthcoming article [26] we consider the problem of the rotational resonance peakshapes of all- $E-\left[11,20-{ }^{13} \mathrm{C}_{2}\right]$-retinal at the higher magnetic field of $9.4 \mathrm{~T}$. We show that the anomalous peakshapes at high field may be explained in terms of the differential transverse relaxation of spins in the two sites. The transverse relaxation differential is due to imperfect heteronuclear decoupling, caused by an interference of the magicangle sample rotation and the proton decoupler field. A revised theory of rotational resonance peakshapes, capable of accommodating such effects, is presented [26].

The same labelled compounds which give discrepancies between theory and experiments at a field of 9.4 T provide "normal" rotational resonance spectra at a lower magnetic field of $4.7 \mathrm{~T}$. In this article, we present a study of the rotational resonance spectrum of one of these compounds, polycrystalline all- $E-\left[11,20-{ }^{13} \mathrm{C}_{2}\right]$-retinal, at the lower field of $4.7 \mathrm{~T}$. As shown below, the rotational resonance peakshapes and magnetization exchange curves may be simulated quite well at this lower field, using the "conventional" theory, as presented in Ref. [3].

\section{Materials}

This study employed three different samples of polycrystalline all-E-retinal (Fig. 1).

(1) Natural abundance all-E-retinal, purchased commercially (Fluka, Switzerland) and used without further purification. We refer to this sample as natRET.

(2) All-E-retinal labelled to a level of larger than $99 \%$ with ${ }^{13} \mathrm{C}$ at both positions $\mathrm{C} 11$ and $\mathrm{C} 20$. This was synthesized according to published procedures [27] and crystallized at $-20^{\circ} \mathrm{C}$ from $n$-pentane. We refer to this sample as $99 \%-{ }^{13} \mathrm{C}_{2}$-RET. 


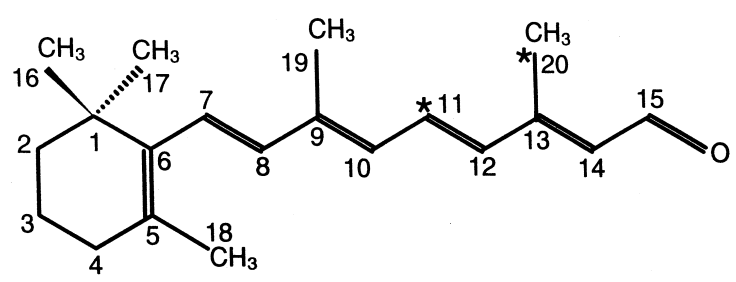

Fig. 1. Numbering scheme of the all-E-retinal molecule. Asterisks denote the ${ }^{13} \mathrm{C}$ labelled sites.

(3) A solid solution of $99 \%-{ }^{13} \mathrm{C}_{2}$-RET in nat-RET, at a molar ratio of 15:100. This sample was prepared by dissolving the appropriate amounts of $99 \%-{ }^{13} \mathrm{C}_{2}$ RET and nat-RET in $n$-pentane and crystallizing. We refer to this sample as $15 \%-{ }^{13} \mathrm{C}_{2}$-RET.

Neighboring all-E-retinal molecules in the crystalline solid have an antiparallel orientation, as determined by X-ray diffraction [28]. The internuclear distance between the $\mathrm{C} 11$ and $\mathrm{C} 20$ sites within the same molecule is $0.296 \pm 0.001 \mathrm{~nm}$. The shortest distances between ${ }^{13} \mathrm{C}$ labels on neighboring molecules are as follows: $r_{11,11^{\prime}}=0.384 \mathrm{~nm} ; r_{11,20^{\prime}}$ $=0.458 \mathrm{~nm} ; r_{20,20^{\prime}}=0.573 \mathrm{~nm}$. In most of the spectra discussed below, we employed the $15 \%$ ${ }^{13} \mathrm{C}_{2}$-RET sample in order to reduce the probability of the intermolecular spin-spin interactions.

\section{Methods}

\subsection{MAS spectra}

${ }^{13} \mathrm{C}$ MAS spectra were recorded at a field strength $B_{0}=4.7 \mathrm{~T}\left({ }^{13} \mathrm{C}\right.$ Larmor frequency $\left.=-50.323 \mathrm{MHz}\right)$ using the standard CP-MAS pulse scheme shown in Fig. 2a. The ${ }^{13} \mathrm{C}$ transverse magnetization was enhanced by ramped cross-polarization (CP) from protons [29-31]. The proton $\pi / 2$ pulse duration was $4.7 \mu \mathrm{s}$. The proton decoupling field strength during data acquisition corresponded to a nutation frequency of $\left|\omega_{\text {nut }}^{\mathrm{I}} / 2 \pi\right| \approx 96 \mathrm{kHz}$.

The rotational resonance spectra were taken for a variety of orders $n$, chosen by adjusting the spinning frequency to the appropriate condition. The relevant spinning frequencies in the field of $B_{0}=4.7 \mathrm{~T}$ were $\left|\omega_{\mathrm{r}} / 2 \pi\right|=6.098 \mathrm{kHz}$ for $n=1 \mathrm{RR},\left|\omega_{\mathrm{r}} / 2 \pi\right|=$ $3.049 \mathrm{kHz}$ for $n=2 \mathrm{RR}$, and $\left|\omega_{\mathrm{r}} / 2 \pi\right|=2.033 \mathrm{kHz}$ for $n=3 \mathrm{RR}$.

\subsection{Zeeman magnetization exchange}

The Zeeman magnetization exchange between the labelled ${ }^{13} \mathrm{C}$ sites was followed using the pulse scheme shown in Fig. 2b. The first part of this pulse scheme (up to and including the first $\pi / 2{ }^{13} \mathrm{C}$ pulse) creates a spin density operator $\rho$ containing a term proportional to the difference angular momentum operator $S_{j z}-S_{k z}$, where $j$ and $k$ are the two labelled spin sites. This is done by allowing the crosspolarized ${ }^{13} \mathrm{C}$ magnetization to evolve under the isotropic shift difference of the two sites. Four $\pi$ pulses are applied at carefully timed intervals so as to suppress the effect of chemical shift anisotropy, as described in Ref. [32]. A $\pi / 2$ pulse of suitable phase converts the out-of-phase transverse magnetization into differential longitudinal magnetization. The longitudinal difference magnetization created by the first $\pi / 2$ pulse is allowed to evolve under the interval $\tau$. Another strong $\pi / 2$ pulse reconverts the Zeeman magnetization into transverse magnetization, which generates an NMR signal in the following interval. A standard phase cycling scheme is used to select signal components passing through longitudi-
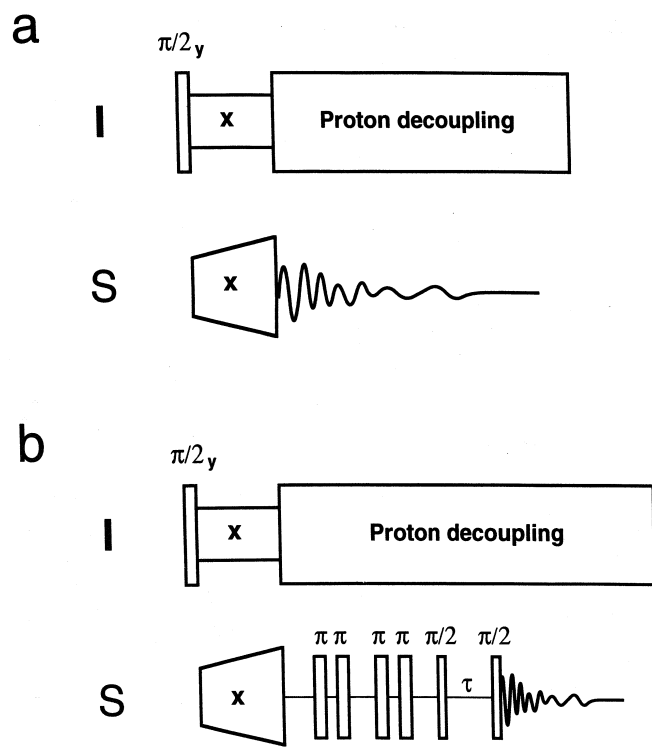

Fig. 2. The $\mathrm{rf}$ pulse scheme for rotational resonance spectral peakshape measurements (a), and for the Zeeman magnetization exchange measurements (b). The ramp of the cross polarization field and switching of the decoupler irradiation amplitude are optional. 
nal magnetization during the $\tau$ interval. After Fourier transformation, the integrated intensities of the two peaks are taken to be proportional to the Zeeman magnetizations of the appropriate sites at the end of the $\tau$ interval. The rotor-driven coherence transfer signals during the acquisition period are known not to affect peak integrals [26]. The experiment is repeated for a set of $\tau$ values, typically ranging from 0 to $50 \mathrm{~ms}$. The difference between integrated peak amplitudes as a function of $\tau$ corresponds to the time course of difference Zeeman magnetization $\left\langle S_{1 \mathrm{z}}-\right.$ $\left.S_{2 z}\right\rangle(\tau)$. The data set is usually normalized so that the first point has unit amplitude.

The experimental Zeeman magnetization exchange data were collected with ${ }^{1} \mathrm{H}$ and ${ }^{13} \mathrm{C} \pi / 2$ pulse durations of 2.3 and $2.4 \mu \mathrm{s}$, respectively. In the results shown, the same rf field was used throughout the proton channel, corresponding to a nutation frequency of $\left|\omega_{\text {nut }}^{\mathrm{I}} / 2 \pi\right| \approx 108 \mathrm{kHz}$.

All experimental data were obtained on a modified Bruker MSL 200 spectrometer at a magnetic field of $4.7 \mathrm{~T}$. A standard Bruker double bearing 4 $\mathrm{mm}$ rotor system $\left(\mathrm{ZrO}_{2}\right.$ rotors, Kel-F end caps) was used. The sample spinning frequency was stabilized to $\pm 2 \mathrm{~Hz}$ using a home-built stabilization device. The CP contact interval was $2 \mathrm{~ms}$.

\subsection{Simulations}

Numerical simulations of rotational resonance spectra and Zeeman magnetization exchange were performed using previously described procedures [3]. The calculations require the following parameters: (i) The principal values of the chemical shift tensors of the two sites; (ii) the isotropic $J$-coupling between the two spins; (iii) the through-space dipolar coupling between the spins; (iv) the relative orientations of the chemical shift tensors with respect to the internuclear vector; (v) relaxation parameters (see below).

The anisotropies of the chemical shift tensors have been derived from an analysis of the slow-spinning MAS spectrum [25]. The values used in the simulations are shown in Table 1 . The chemical shift anisotropy (in deshielding units) is defined $\delta_{\text {aniso }}^{j}=$ $\delta_{z z}^{j}-\delta_{\text {iso }}^{j}$, where $\delta_{\text {iso }}^{j}$ is the isotropic chemical shift and the principal values are ordered as $\left|\delta_{z z}^{j}-\delta_{\text {iso }}^{j}\right|>$ $\left|\delta_{x x}^{j}-\delta_{\text {iso }}^{j}\right|>\left|\delta_{y y}^{j}-\delta_{\text {iso }}^{j}\right|$ [35]. The asymmetry pa-
Table 1

Parameters for the rotational resonance simulations of all- $E$ $\left[11,20-{ }^{13} \mathrm{C}_{2}\right]$-retinal, at a magnetic field of $B_{0}=4.7 \mathrm{~T}$

\begin{tabular}{lll}
\hline Parameters & $\begin{array}{l}\text { all- } E-\left[11,20-{ }^{13} \mathrm{C}_{2}\right]- \\
\text { retinal }\end{array}$ & Notes \\
\hline$\left(\delta_{\text {iso }}^{j}-\delta_{\text {iso }}^{k}\right)[\mathrm{ppm}]$ & -121.2 & $\mathrm{a}$ \\
$\delta_{\text {aniso }}^{k}[\mathrm{ppm}]$ & 112.2 & $\mathrm{~b}$ \\
$\eta^{k}$ & 0.72 & $\mathrm{~b}$ \\
$\left\{\alpha_{\mathrm{PM}}^{k}, \beta_{\mathrm{PM}}^{k}, \gamma_{\mathrm{PM}}^{k}\right\}$ & $\{-31,85,-87\}$ & $\mathrm{c}$ \\
$\delta_{\text {aniso }}^{j}[\mathrm{ppm}]$ & 15.5 & $\mathrm{~b}$ \\
$\eta^{j}$ & 1.0 & $\mathrm{~b}$ \\
$\left\{\alpha_{\mathrm{PM}}^{j}, \beta_{\mathrm{PM}}^{j}, \gamma_{\mathrm{PM}}^{j}\right\}$ & $\{0,57,0\}$ & $\mathrm{c}$ \\
$(b / 2 \pi)[\mathrm{Hz}]$ & -293 & $\mathrm{~d}$ \\
$J[\mathrm{~Hz}]$ & 0 & $\mathrm{e}$ \\
$T_{2}^{\mathrm{SQ}}[\mathrm{ms}]$ & 15.9 & $\mathrm{f}$ \\
$T_{2}^{\mathrm{ZQ}}[\mathrm{ms}]$ & 11.0 & $\mathrm{~g}$ \\
Estimated internuclear & & \\
distance $(\mathrm{nm})$ & & \\
from RR spectra & $0.301 \pm 0.008 \mathrm{~nm}$ & $\mathrm{~h}$ \\
from RR magnetization & $0.300 \pm 0.010 \mathrm{~nm}$ & $\mathrm{i}$ \\
$\quad$ exchange & & \\
from X-ray diffraction & $0.296 \pm 0.001 \mathrm{~nm}$ & \\
\hline
\end{tabular}

(a) Difference in isotropic chemical shifts (deshielding units) between the $\mathrm{C} 20$ site (index $j$ ) and $\mathrm{C} 11$ site (index $k$ ).

(b) Chemical shift anisotropy parameters (deshielding units) for the $\mathrm{C} 11$ and $\mathrm{C} 20$ sites, as reported in Ref. [25].

(c) Euler angles in degrees, defining the assumed chemical shift tensor orientations with respect to the molecular reference frame (chosen such that the $z$-axis is along the vector joining the $\mathrm{C} 11$ and $\mathrm{C} 20$ nuclei and the $x$-axis is in the $(\mathrm{C} 11, \mathrm{C} 12, \mathrm{C} 20)$ plane.

(d) Dipole-dipole coupling constant from the X-ray structure $r=0.296 \mathrm{~nm}$.

(e) The three-bond ${ }^{13} \mathrm{C}-{ }^{13} \mathrm{C} J$ coupling is ignored.

(f) Single-quantum relaxation time constant estimated from the mean of the off-rotational resonance peak widths, at a proton decoupling field corresponding to $\left|\omega_{\text {nut }}^{\mathrm{I}} / 2 \pi\right| \approx 96 \mathrm{kHz}$.

(g) Zero-quantum relaxation time constant estimated from the single-quantum spectral peak widths, at a proton decoupling field corresponding to $\left|\omega_{\text {nut }}^{\mathrm{I}} / 2 \pi\right| \approx 108 \mathrm{kHz}$.

(h) Estimated internuclear distance from rotational resonance spectra of the $15 \%{ }^{13} \mathrm{C}_{2}$-labelled sample.

(i) Estimated internuclear distance from rotor-driven Zeeman magnetization exchange on the $99 \%{ }^{13} \mathrm{C}_{2}$-labelled sample.

rameter $\eta^{j}$ of the chemical shift tensor is defined through $\eta^{j}=\left(\delta_{y y}^{j}-\delta_{x x}^{j}\right) / \delta_{\text {aniso }}^{j}$. The superscripts $j$ and $k$ denote the $\mathrm{C} 20$ and $\mathrm{C} 11$ sites, respectively. The isotropic chemical shift frequency difference is given by $\omega_{\Delta}^{\text {iso }}=\omega_{\text {iso }}^{j}-\omega_{\text {iso }}^{k}=\omega_{0}\left(\delta_{\text {iso }}^{j}-\delta_{\text {iso }}^{k}\right)$, where $\omega_{0}$ is the signed Larmor frequency $\omega_{0}=-\gamma B_{0}$ [36].

The Euler angles $\left\{\alpha_{\mathrm{PM}}^{j}, \beta_{\mathrm{PM}}^{j}, \gamma_{\mathrm{PM}}^{j}\right\}$ define the relative orientation of the chemical shift tensor principal axis system $\mathrm{P}$ of site $j$ and a molecular axis system $M$ whose $z$-axis is defined as the internuclear 
C11-C20 vector. The Euler angles are defined as in Ref. [37]. The Euler angles used in the simulation were calculated from the crystal structure geometry combined with plausible assumptions about the orientations of the chemical shift tensors with respect to the local functional groups. The principal axis system of the $\mathrm{C} 11$ site is defined such that the most shielded element is perpendicular to the (C10, C11, C12) plane, while the intermediate shielded element is along the $\mathrm{C} 11-\mathrm{C} 12$ bond. The orientation of the principal axis system of $\mathrm{C} 20$ site is defined so that the most shielded element is along the $\mathrm{C} 13-\mathrm{C} 20$ bond and the least shielded one is in the $(\mathrm{C} 12, \mathrm{C} 13$, C20) plane. These orientations conform to the consensus of single crystal studies in related compounds [38]. It should be emphasized that, at least for the $n=1 \mathrm{RR}$, the simulated behavior is insensitive to these chemical shift tensor orientations [25].

The three-bond $J$-coupling between the $\mathrm{C} 11$ and C20 sites was assumed to be negligible.

The rotational resonance peakshapes were simulated by evaluating the dynamics of the isolated spin-pair system, ignoring the transverse relaxation of the magnetization components. The simulated free induction decay was multiplied by an exponential damping function to take into account transverse relaxation effects [3]. This procedure is justifiable if the transverse relaxation time constants are the same for both sites [26]. The observed peak widths (full widths at half height) were $\sim 20 \mathrm{~Hz}$ for both the C11 site and the C20 sites at a spinning frequency of $\left|\omega_{\mathrm{r}} / 2 \pi\right|=10.5 \mathrm{kHz}$ and a decoupler field strength corresponding to the proton nutation frequency $\left|\omega_{\text {nut }}^{\mathrm{I}} / 2 \pi\right| \approx 96 \mathrm{kHz}$.

The magnetization exchange simulations require estimations of the zero-quantum relaxation time constant $T_{2}^{\mathrm{ZQ}}$. This parameter was estimated by making a further assumption that the transverse relaxation is caused by uncorrelated fluctuating random fields at the two sites. The zero-quantum relaxation rate constant is then equal to the sum of the single-quantum relaxation rate constants of the two sites [3]. In the present case, the peak widths (full-width-at-halfmaximum) of $14 \mathrm{~Hz}$ for the $\mathrm{C} 11$ site and $15 \mathrm{~Hz}$ for the C20 site at $\left|\omega_{\mathrm{r}} / 2 \pi\right|=10 \mathrm{kHz}$ and $\left|\omega_{\text {nut }}^{\mathrm{I}} / 2 \pi\right| \approx$ $108 \mathrm{kHz}$ allowed the estimate $T_{2}^{\mathrm{ZQ}}=(\pi(14+15))^{-1}$ $=11.0 \mathrm{~ms}$. The simulations are insensitive to this parameter in the regime discussed here.

\section{Results and discussion}

\subsection{Rotational resonance peakshapes}

Expanded regions of the MAS spectrum of natRET at a spinning frequency $\left|\omega_{\mathrm{r}} / 2 \pi\right|=6.098 \mathrm{kHz}$ are shown in Fig. 3a. Most of the peaks may be assigned to the ${ }^{13} \mathrm{C}$ sites according to the solutionstate chemical shifts reported in Ref. [33], and the solid-state data reported in Ref. [34].

The data in Refs. [33,34] indicate nearly coincident ${ }^{13} \mathrm{C}$ chemical shifts for the $\mathrm{C} 19$ and $\mathrm{C} 20$ sites, and for the $\mathrm{C} 11, \mathrm{C} 12$, and $\mathrm{C} 6$ sites. The measured shift frequency difference between the $\mathrm{C} 11$ and $\mathrm{C} 20$
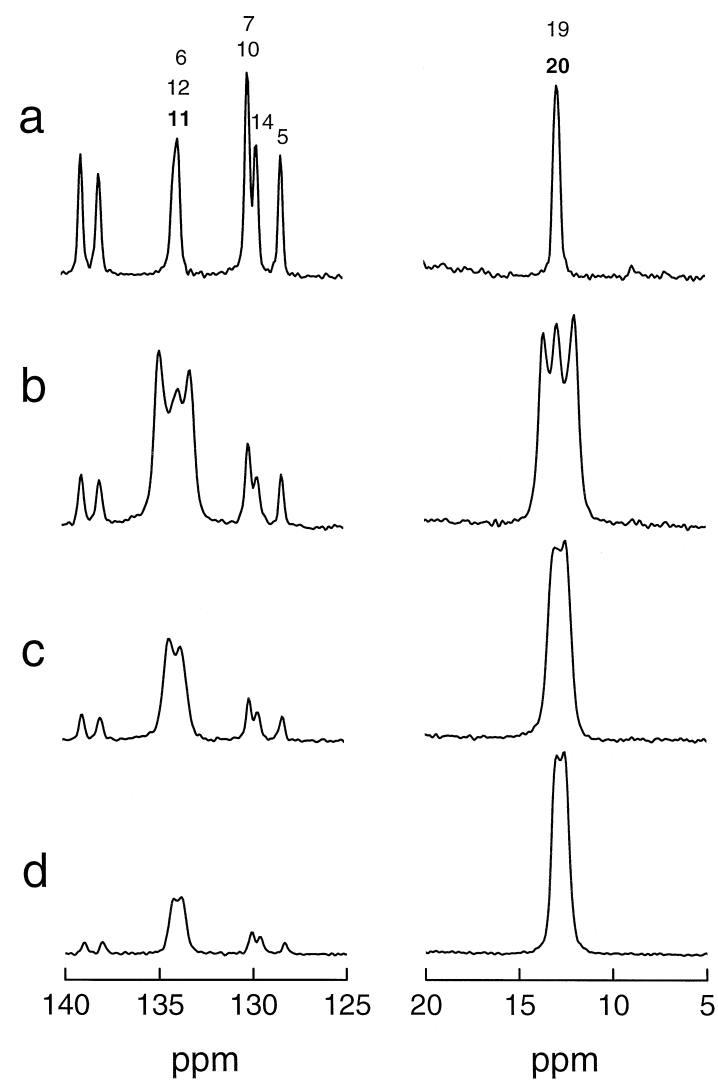

Fig. 3. Expanded regions around the isotropic chemical shifts of the $\mathrm{C} 11$ (left) and $\mathrm{C} 20$ (right) sites of the MAS spectra of all-E-retinal. (a) Natural abundance retinal, at a spinning frequency of $\left|\omega_{\mathrm{r}} / 2 \pi\right|=6.098 \mathrm{kHz}$. (b) $15 \%{ }^{-13} \mathrm{C}_{2}$-RET at the $n=1$ RR condition $\left(\left|\omega_{\mathrm{r}} / 2 \pi\right|=6.098 \mathrm{kHz}\right)$; (c) $15 \%-{ }^{13} \mathrm{C}_{2}$-RET at the $n=2$ RR condition $\left(\left|\omega_{\mathrm{r}} / 2 \pi\right|=3.049 \mathrm{kHz}\right)$; (d) $15 \%-{ }^{13} \mathrm{C}_{2}-\mathrm{RET}$ at the $n=3 \mathrm{RR}$ condition $\left(\left|\omega_{\mathrm{r}} / 2 \pi\right|=2.033 \mathrm{kHz}\right)$. 
peaks in nat-RET is $\omega_{\Delta}^{\text {iso }} / 2 \pi=6.088 \mathrm{kHz}$ at a field of $4.7 \mathrm{~T}$.

The MAS spectrum of the $15 \%-{ }^{13} \mathrm{C}_{2}$-RET sample, at the $n=1$ rotational resonance $\left(\left|\omega_{\mathrm{r}} / 2 \pi\right|=\right.$ $6.098 \mathrm{kHz})$ is shown in Fig. 3b. The prominent spectral features come from the ${ }^{13} \mathrm{C}$ labels at the $\mathrm{C} 11$ and $\mathrm{C} 20$ sites, broadened by the dipole-dipole coupling at the $n=1$ rotational resonance condition. Spectra at the $n=2$ rotational resonance $\left(\left|\omega_{\mathrm{r}} / 2 \pi\right|\right.$ $=3.049 \mathrm{kHz})$ and the $n=3$ rotational resonance $\left(\left|\omega_{\mathrm{r}} / 2 \pi\right|=2.033 \mathrm{kHz}\right)$ are shown in Fig. 3c and $\mathrm{d}$.

The NMR signals from the $15 \%-{ }^{13} \mathrm{C}_{2}$-RET sample come from three major classes of molecules: (i) molecules which have introduced ${ }^{13} \mathrm{C}$ labels at both sites $\mathrm{C} 11$ and $\mathrm{C} 20$, and no naturally occurring ${ }^{13} \mathrm{C}$ spins; (ii) molecules with no introduced ${ }^{13} \mathrm{C}$ labels but a singly naturally occurring ${ }^{13} \mathrm{C}$ spin; (iii) molecules containing a natural ${ }^{13} \mathrm{C}$ spin as well as two artificial ${ }^{13} \mathrm{C}$ labels. As discussed in Ref. [26], the signal contributions from class (ii) and (iii) molecules may be eliminated, to a good approximation, by subtracting a suitably weighted spectrum of nat-RET, obtained under identical experimental conditions. Such "difference spectra" at the $n=1,2,3$ RR conditions are shown in Fig. 4. Simulations using the parameters in Table 1 are also shown. The measured and simulated rotational resonance peakshapes are in very good agreement. There are minor deviations at $n=2$ and $n=3 \mathrm{RR}$ (the experimental inner peak amplitudes being smaller than the simulated amplitudes). This may reflect a small change $(\sim 10 \mathrm{~Hz})$ of the isotropic ${ }^{13} \mathrm{C}$ chemical shift in the ${ }^{13} \mathrm{C}_{2}$-labelled molecules, due to the influence of the second ${ }^{13} \mathrm{C}$ on the molecular vibrations and librations. Such effects are frequently observed in solution NMR $[39,40]$.

To reveal the resolution of the determined distance by the rotational resonance peakshapes, a series of peakshape simulations at $n=1 \mathrm{RR}$ varying only the dipole-dipole coupling constant was carried out. Some results are shown in Fig. 5. The best overall match is for a dipole-dipole coupling constant $b / 2 \pi=-280 \mathrm{~Hz}$, corresponding to an internuclear distance $r=0.301 \mathrm{~nm}$. A $20 \mathrm{~Hz}$ deviation of the dipole-dipole coupling constant may easily be resolved. This translates into a rough confidence limit in the internuclear distance of $\pm 0.008 \mathrm{~nm}$. The $\mathrm{X}$-ray estimation of $r=0.296 \mathrm{~nm}$ is within this a
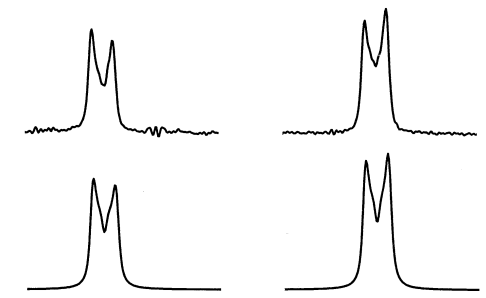

b
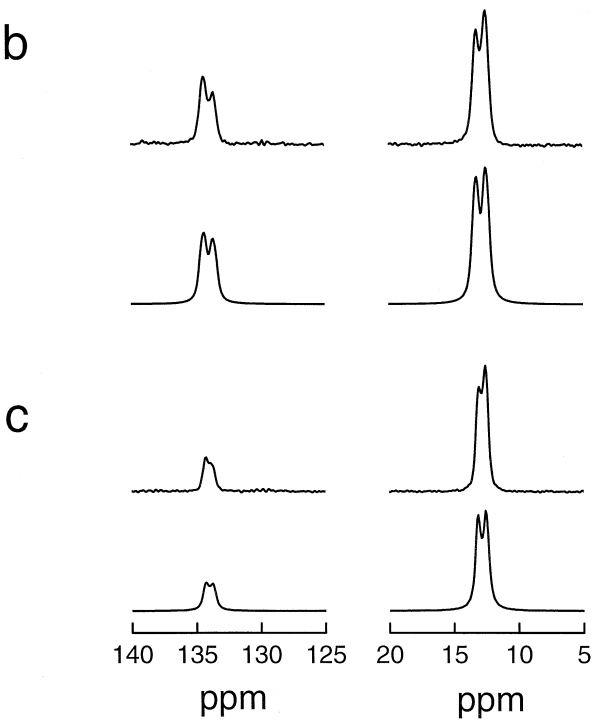

Fig. 4. Expanded regions around the isotropic chemical shifts of the $\mathrm{C} 11$ (left) and $\mathrm{C} 20$ (right) sites at $n=1,2$, and $3 \mathrm{RR}$ conditions ((a), (b), and (c) respectively). At each RR condition, the upper figures represent experimental rotational resonance spectra of $15 \%-{ }^{13} \mathrm{C}_{2}$-RET (with natural abundance spectra subtracted, see text); the lower figures are simulations using the parameters listed in Table 1.

confidence limit. A more rigorous estimation of the confidence limits could be obtained by statistical $\chi^{2}$ analysis [41].

To illustrate the effect of the intermolecular interactions on the rotational resonance peakshapes, spectra were also recorded on the $99 \%-{ }^{13} \mathrm{C}_{2}$-RET sample at $n=1,2$, and 3 rotational resonances. Expanded regions around the isotropic chemical shifts of the C11 and C20 sites are displayed in Fig. 6.

At $n=1 \mathrm{RR}$, the multiplets have a similar appearance as for the $15 \%$ doubly labelled sample. In particular, the $\mathrm{C} 20$ peak displays a prominent central feature in both the $15 \%-{ }^{13} \mathrm{C}_{2}$-RET and $99 \%-{ }^{13} \mathrm{C}_{2}$ RET sample. However, the origin of the central 
a
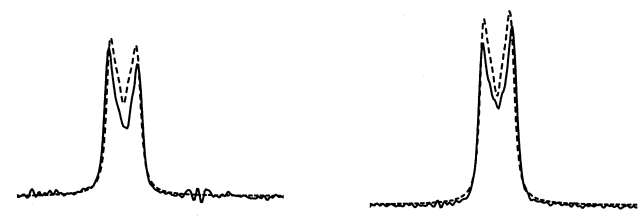

b
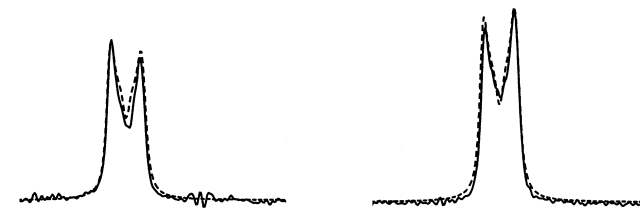

C
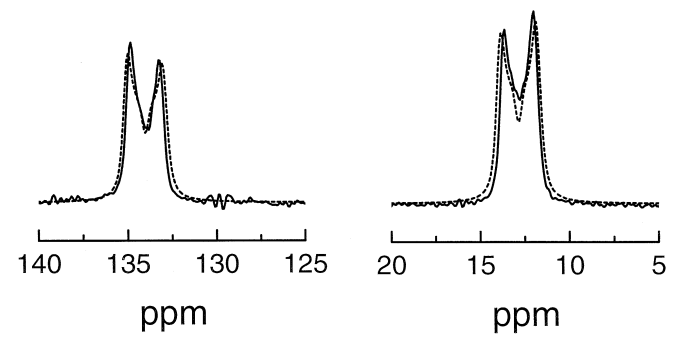

Fig. 5. Expanded regions around the isotropic chemical shifts of the $\mathrm{C} 11$ site (left) and the $\mathrm{C} 20$ site (right) of the $n=1$ rotational resonance difference spectra, compared with simulated spectra for the following values of dipole-dipole coupling constant $b / 2 \pi$ : (a) $-253 \mathrm{~Hz}$, (b) $-293 \mathrm{~Hz}$, (c) $-333 \mathrm{~Hz}$. Solid lines represent the experimental spectra; dashed lines represent simulations.

feature in the $\mathrm{C} 20$ peak must be different in these two materials. In $15 \%-{ }^{13} \mathrm{C}_{2}$-RET, this peak could be attributed to isotopomers containing isolated ${ }^{13} \mathrm{C}$ spins. Subtraction of the natural abundance spectrum essentially eliminates this peak. In the $99 \%-{ }^{13} \mathrm{C}_{2}$-RET sample, on the other hand, the singly ${ }^{13} \mathrm{C}$-labelled isotopomers are quite rare, arising mainly from incomplete labelling of the carbon sites in the starting materials. These isotopomers are too rare to account for the central feature in the C20 peak. Signals from ${ }^{13} \mathrm{C}_{3}$ isotopomers also contribute, but the relevant signals are severely broadened by rotational resonance effects. For example, consider the 11,19,20${ }^{13} \mathrm{C}_{3}$ isotopomer. Since the isotropic chemical shifts of the $\mathrm{C} 19$ and $\mathrm{C} 20$ sites are almost coincident, and the $\mathrm{C} 11 \ldots \mathrm{C} 20$ and $\mathrm{C} 11 \ldots \mathrm{C} 19$ distances are comparable, the three-spin system in $11,19,20-{ }^{13} \mathrm{C}_{3}$-reti- nal is at "double rotational resonance" and gives broad signals for all sites. These broad signals cannot be responsible for the sharp central feature in the C20 peak.

Intermolecular spin-spin interactions provide an alternative explanation of the central feature in the C20 peak of the $99 \%-{ }^{13} \mathrm{C}_{2}$ labelled material. As remarked above, $\mathrm{C} 11$ sites on neighbouring molecules are rather close $(0.384 \mathrm{~nm})$. The strong intermolecular interactions of the $\mathrm{C} 11$ sites lead to enhanced transverse relaxation for spins in those sites. This enhanced transverse relaxation has an indirect decoupling effect on the C20 resonances, as described in Ref. [26]. Loosely speaking, the enhanced relaxation of the $\mathrm{C} 11$ coherences takes those spins out of rotational resonance, and narrows the C20 signals.

The $\mathrm{C} 11$ multiplet in the $99 \%-{ }^{13} \mathrm{C}_{2}$-RET spectrum at $n=1 \mathrm{RR}$ also displays a sharp central feature. This is probably also due, at least in part, to inter-
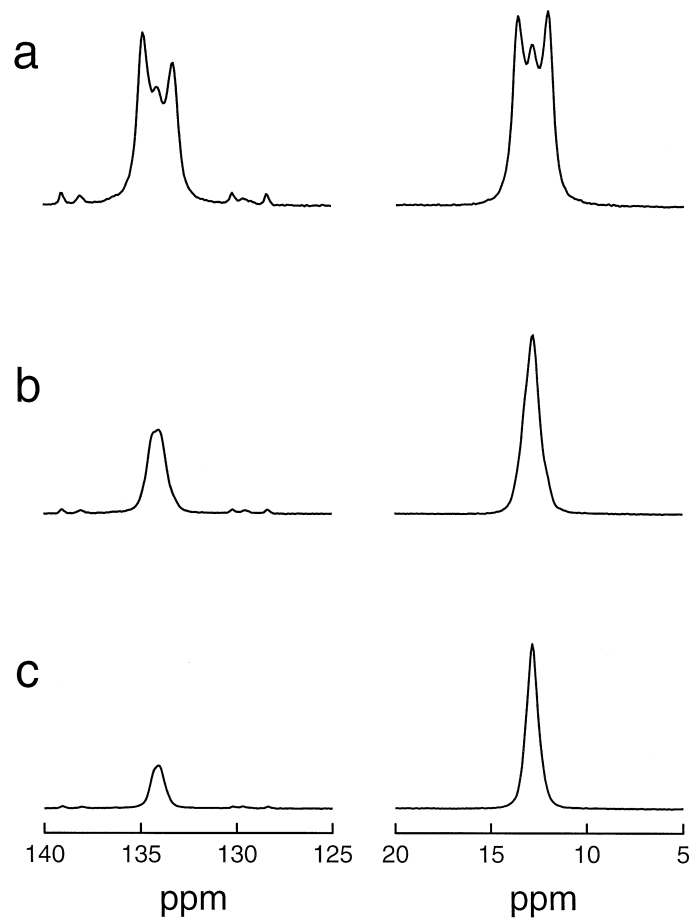

Fig. 6. Expanded regions around the isotropic chemical shifts of the $\mathrm{C} 11$ (left) and $\mathrm{C} 20$ sites of the rotational resonance spectra for the $99 \%-{ }^{13} \mathrm{C}_{2}$-RET sample at (a) $n=1$, (b) $n=2$, and (c) $n=3$ RR conditions. 
molecular spin-spin interactions. Signals from the $11,12,20-{ }^{13} \mathrm{C}_{3}$ isotopomer may also contribute.

Intermolecular interaction effects on the rotational resonance peakshapes may also be noticed clearly at the $n=2$ and 3 rotational resonance. The small splittings observed in the peaks of $15 \%-{ }^{13} \mathrm{C}_{2}$-RET vanish for the case of $99 \%-{ }^{13} \mathrm{C}_{2}$-RET sample. Notice that the $\mathrm{C} 20$ peaks in $99 \%-{ }^{13} \mathrm{C}_{2}$-RET are narrower than in $15 \%-{ }^{13} \mathrm{C}_{2}$-RET. This is consistent with the "self-decoupling', mechanism indicated above, which is discussed in detail in Ref. [26].

\subsection{Zeeman magnetization exchange}

Experimental Zeeman magnetization exchange curves for $99 \%-{ }^{13} \mathrm{C}_{2}$-RET, at $n=1,2$, and $3 \mathrm{RR}$, as well as simulated curves, are shown in Fig. 7. The dipole-dipole coupling constant used in the simula-

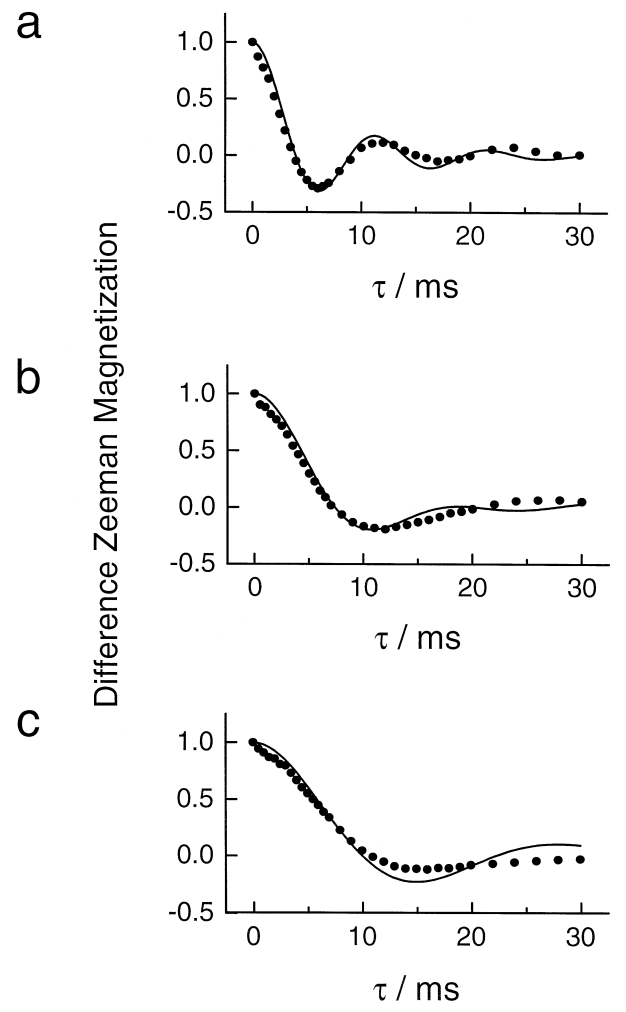

Fig. 7. Experimental and simulated Zeeman magnetization exchange curves for $99 \%-{ }^{13} \mathrm{C}_{2}$-RET sample at (a) $n=1$, (b) $n=2$, and (c) $n=3$ RR. Filled circles represent experimental data, solid lines indicate simulations.
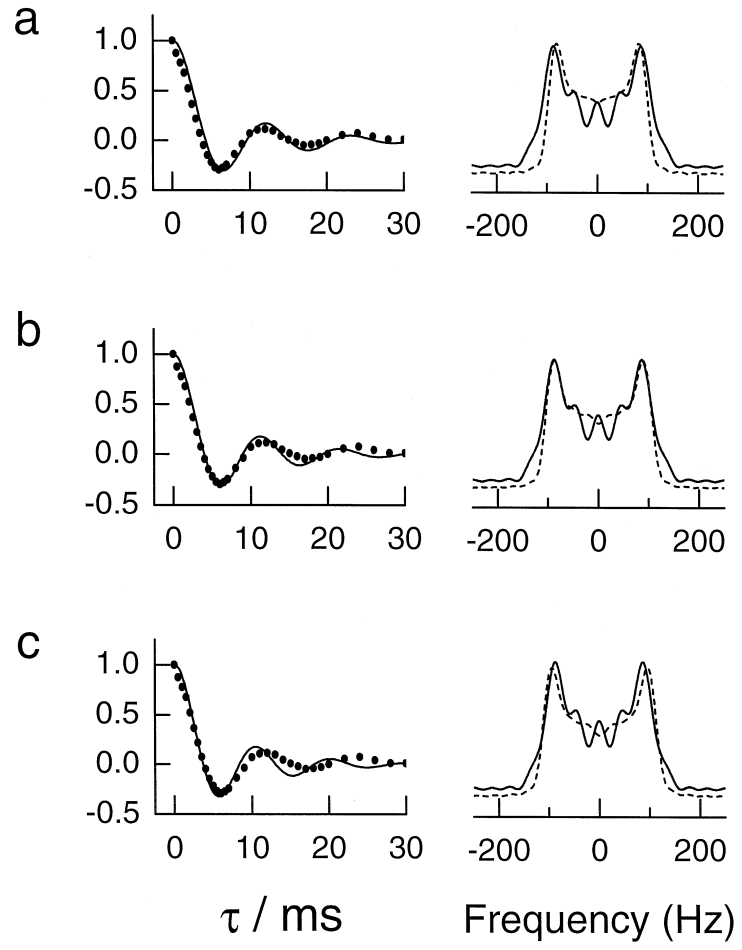

Fig. 8. Time-domain (left) and frequency-domain (right) experimental and simulated Zeeman magnetization exchange curves at the $n=1$ rotational resonance condition. Simulated curves are shown for the following dipole-dipole coupling constants: (a) $b / 2 \pi=-273 \mathrm{~Hz}$, (b) $b / 2 \pi=-293 \mathrm{~Hz}$, and (c) $b / 2 \pi=-313$ Hz. In the time-domain figures, filled circles represent experimental data, solid lines indicate simulations. In the frequency-domain figures, solid lines represent the experimental data and dashed lines indicate simulations.

tions is $-293 \mathrm{~Hz}$, corresponding to the X-ray internuclear distance of $0.296 \mathrm{~nm}$. Other parameters used are listed in Table 1. The agreement between simulated and measured curves is acceptable, at least with respect to the initial decay. The discrepancies in the form of the curve may reflect the influence of intermolecular ${ }^{13} \mathrm{C}-{ }^{13} \mathrm{C}$ interactions. Note that the $n=1$ curve is insensitive to the tensor orientations, so this cannot be held responsible for the deviations.

To reveal the resolution of the determined distance, a series of simulated curves at $n=1$ for a selection of dipole-dipole coupling constants is shown in Fig. 8. The comparison was made using both the direct time-domain magnetization exchange data (left), as well as Fourier transforms of the 
magnetization exchange curves (right) [42]. The time-domain curves appear to give the subjectively best fit for a through-space coupling of $b / 2 \pi=$ $-273 \mathrm{~Hz}$ (Fig. 8a, left plot). The frequency-domain curves, on the other hand, appear to give the subjectively best fit for a through-space coupling of $b / 2 \pi$ $=-293 \mathrm{~Hz}$, if one concentrates on the outer peak splitting, and ignores the discrepancies in the central part (Fig. 8b, right plot). The dipole-dipole coupling may therefore be estimated from the magnetization exchange curves to be $b / 2 \pi=-283 \pm 30 \mathrm{~Hz}$. This translates into an internuclear distance estimate of $0.300 \pm 0.010 \mathrm{~nm}$, which is consistent with the X-ray diffraction value of $0.296 \pm 0.001 \mathrm{~nm}$. A statistical $\chi^{2}$ analysis [41] would give a more rigorous estimate of the confidence limits.

It is difficult to compare the accuracy of the two methods directly (rotational resonance spectra against rotational resonance magnetization exchange), since the data in Figs. 5 and 8 were obtained on samples with different levels of isotope labelling. Magnetization exchange experiments on the $15 \%-{ }^{13} \mathrm{C}_{2}$-RET sample would clarify this issue.

\section{Conclusions}

The internuclear distance between the ${ }^{13} \mathrm{C}$-labelled sites in all- $E-\left[11,20-{ }^{13} \mathrm{C}_{2}\right]$-retinal was estimated using rotational resonance spectra and rotor-driven longitudinal magnetization exchange, in a magnetic field of $B_{0}=4.7 \mathrm{~T}$. The estimated distances of $0.301 \pm$ $0.008 \mathrm{~nm}$ (for rotational resonance spectra of a $15 \%$ ${ }^{13} \mathrm{C}_{2}$-labelled sample) and $0.300 \pm 0.010 \mathrm{~nm}$ (for Zeeman magnetization exchange on a $99 \%{ }^{13} \mathrm{C}_{2}$ labelled sample) both agree well with the $\mathrm{X}$-ray crystal structure.

Significant deviations between theoretical and experimental rotational resonance spectra were obtained when the ${ }^{13} \mathrm{C}_{2}$-labelled molecules were not diluted in an unlabelled matrix. These deviations were attributed to intermolecular ${ }^{13} \mathrm{C}-{ }^{13} \mathrm{C}$ interactions.

\section{Acknowledgements}

This research was supported by the Swedish National Science Foundation, the EU Biotechnology program (PL920467) and the Göran Gustafsson Foundation for Research in the Natural Sciences and Medicine. We would like to acknowledge Ole G. Johannessen and Torgny Karlsson for their experimental and computer help.

\section{References}

[1] D.P. Raleigh, M.H. Levitt, R.G. Griffin, Chem. Phys. Lett. 146 (1988) 71.

[2] T. Gullion, J. Schaefer, J. Magn. Reson. 81 (1989) 479.

[3] M.H. Levitt, D.P. Raleigh, F. Creuzet, R.G. Griffin, J. Chem. Phys. 92 (1990) 6347.

[4] D.M. Gregory, D.J. Mitchell, J.A. Stringer, S. Kiihne, J.C. Shiels, J. Callahan, M.A. Mehta, G.P. Drobny, Chem. Phys. Lett. 246 (1995) 654.

[5] A.C. deDios, E. Oldfield, Solid State Nucl. Magn. Reson. 6 (1996) 101

[6] M. Han, S.O. Smith, Biochemistry 34 (1995) 1425.

[7] Y. Tomita, E.J. O'Connor, A. McDermott, J. Am. Chem. Soc. 116 (1994) 8766.

[8] D.P. Weliky, R. Tycko, J. Am. Chem. Soc. 118 (1996) 8487.

[9] K. Schmidt-Rohr, J. Am. Chem. Soc. 118 (1996) 7601.

[10] X. Feng, Y.K. Lee, D. Sandström, M. Edén, H. Maisel, A. Sebald, M.H. Levitt, Chem. Phys. Lett. 257 (1996) 314.

[11] E.R. Andrew, A. Bradbury, R.G. Eades, V.T. Wynn, Phys. Lett. 4 (1963) 99.

[12] J. Heller, R. Larsen, M. Ernst, A.C. Kolbert, M. Baldwin, S.B. Prusiner, D.E. Wemmer, A. Pines, Chem. Phys. Lett. 251 (1996) 223.

[13] A. McDermott, F. Creuzet, R.G. Griffin, Biochemistry 29 (1990) 5767.

[14] F. Creuzet, A. McDermott, R. Gebhard, K. van der Hoff, M.B. Spijker-Assink, J. Herzfeld, M.H. Levitt, R.G. Griffin, Science 251 (1991) 783.

[15] O.B. Peersen, S. Yoshimura, H. Hojo, S. Aimoto, S.O. Smith, J. Am. Chem. Soc. 114 (1992) 4332.

[16] O.B. Peersen, S.O. Smith, Concepts Magn. Reson. 5 (1993) 303.

[17] K.V. Lakshmi, M. Auger, J. Raap, J. Lugtenburg, R.G. Griffin, J. Herzfeld, J. Am. Chem. Soc. 115 (1993) 8515.

[18] A. McDermott, F. Creuzet, R. Gebhard, K. van der Hoef, M.H. Levitt, J. Herzfeld, J. Lugtenburg, R.G. Griffin, Biochemistry 33 (1994) 6129.

[19] S.O. Smith, J. Hamilton, A. Salmon, B.J. Bormann, Biochemistry 33 (1994) 6327.

[20] S.O. Smith, R. Jonas, M. Braiman, B.J. Bormann, Biochemistry 33 (1994) 6334.

[21] J.M. Griffiths, T.T. Ashburn, M. Auger, P.R. Costa, R.G. Griffin, P.T. Lansbury, J. Am. Chem. Soc. 117 (1995) 3539.

[22] O.B. Peersen, M. Groesbeek, S. Aimoto, S.O. Smith, J. Am. Chem. Soc. 117 (1995) 7228.

[23] S.O. Smith, B.J. Bormann, Proc. Natl. Acad. Sci. U.S.A. 92 (1995) 488 
[24] P.T. Lansbury, P.R. Costa, J.M. Griffiths, E.J. Simon, M. Auger, K.J. Halverson, D.A. Kocisko, Z.S. Hendsch, T.T. Ashburn, R.G.S. Spencer, B. Tidor, R.G. Griffin, Nat. Struct. Biol. 2 (1995) 990.

[25] P.J.E. Verdegem, M. Helmle, J. Lugtenburg, H.J.M. de Groot, J. Am. Chem. Soc. 119 (1997) 169.

[26] M. Helmle, Y.K. Lee, P.J.E. Verdegem, T. Karlsson, H.J.M. de Groot, J. Lugtenburg, M.H. Levitt, submitted for publication.

[27] M. Groesbeek, J. Lugtenburg, J. Photochem. Photobiol. 56 (1992) 903.

[28] T. Hamanaka, T. Mitsui, Acta Crystallogr., Sect. B 28 (1972) 214.

[29] S. Zhang, C.L. Czekaj, W.T. Ford, J. Magn. Reson. A 111 (1994) 87.

[30] G. Metz, X. Wu, S.O. Smith, J. Magn. Reson. A 110 (1994) 219.

[31] S. Hediger, B.H. Meier, N.D. Kurur, G. Bodenhausen, R.R. Ernst, Chem. Phys. Lett. 223 (1994) 283.

[32] H. Geen, M.H. Levitt, G. Bodenhausen, Chem. Phys. Lett. 200 (1992) 350.
[33] G. Englert, Helv. Chim. Acta 58 (1975) 2367.

[34] G.S. Harbison, P.P.J. Mulder, H. Pardoen, J. Lugtenburg, J. Herzfeld, R.G. Griffin, J. Am. Chem. Soc. 107 (1985) 4809.

[35] D.M. Grant, in: Encyclopedia of NMR, Vol. 2, Wiley, Chichester, England, 1996.

[36] M.H. Levitt, J. Magn. Reson. 126 (1997) 164.

[37] D.A. Varshalovich, A.N. Moskalev, V.K. Khersonskii, Quantum Theory of Angular Momentum, World Scientific, Singapore, 1988.

[38] M. Mehring, Principles of High Resolution NMR in Solids, 2nd edn., Springer, Berlin, 1983.

[39] H. Batiz-Hernandez, R.A. Bernheim, Prog. NMR Spectrosc. 3 (1967) 63.

[40] C.J. Jameson, in: Encyclopedia of NMR, Vol. 4, Wiley, Chichester, England, 1996.

[41] W.H. Press, B.P. Flannery, S.A. Teukolsky, W.T. Vetterling, Numerical Recipes. The Art of Scientific Computing. Cambridge Univ. Press, Cambridge, 1986.

[42] N.C. Nielsen, F. Creuzet, R.G. Griffin, J. Magn. Reson. A 103 (1993) 245. 